\title{
Viscosity Study on Interaction between Sequence-Ordered Methacrylic Acid Copolymer and Poly(4-vinylpyridine)
}

\author{
Yoshihito InaI, ${ }^{\dagger}$ Sigenobu TaKenOUCHI, Tadamichi HirabaYaSHI, \\ and Kenji YoKota \\ Department of Materials Science and Engineering, Nagoya Institute of Technology, \\ Gokiso-cho, Showa-ku, Nagoya 466, Japan
}

(Received June 20, 1995)

\begin{abstract}
KEY WORDS Viscometry / Polymer Solvent Method / Sequence-Ordered Copolymer / Methacrylic Acid / Poly(4-vinylpyridine) / Hydrogen-Bonding / Hydrophobic Interaction /
\end{abstract}

The viscosity study on ternary system of a lowmolecular-weight solvent and two chemically dissimilar polymers provides useful information about association and complex formation between the two polymers. Such studies have been carried out in homopolymer systems, but have not almost focused on systems containing copolymers, particularly sequence-ordered copolymers.

In the present note, we have investigated interaction between two kinds of acid-(co)polymers $\mathbf{1}-\mathbf{2}$ and a basehomopolymer 3 by viscosity measurement: i.e., poly(methacrylic acid) 1 , periodic $(2: 1)$ ethylene-methacrylic acid copolymer 2 , and poly(4-vinylpyridine) $3 .^{1,2}$<smiles>CCC(C)(C)C(=O)O</smiles><smiles>CCCCCC(C)(C)C(=O)O</smiles>

1<smiles>CCc1ccncc1</smiles>

3

The interaction coefficient $\left(k_{\mathrm{AB}}\right)$ was determined for $\mathbf{1} / \mathbf{3}$ and $2 / 3$ pairs in dimethyl sulfoxide (DMSO). The $k_{\mathrm{AB}}$ value, ${ }^{3,4}$ being similar to Huggins coefficient, originates from a superposition of several types of interactions (e.g., hydrodynamic and thermodynamic interactions), and thus provides information on the interaction for $1 / 3$ and $2 / 3$ pairs.

\section{EXPERIMENTAL}

Preparation of the acid-polymer samples, and their characterization have been previously described. ${ }^{3}$ Polymer 3 was prepared by radical polymerization of 4vinylpyridine with 2,2'-azobisisobutyronitrile. Gel-permeation chromatography was determined in tetrahydrofuran at $313 \mathrm{~K}$ on a Tosoh HLC 803D chromatograph

\footnotetext{
$\uparrow$ To whom correspondence should be addressed.
}

with GMX-, G1000-, G2000-, and G4000-HXL columns in series. Chromatograms were calibrated by polystyrene standards to calculate $M_{n}: 5.6 \times 10^{4}$ for $1,6.6 \times 10^{4}$ for 2, and $2.0 \times 10^{5}$ for 3 . The ${ }^{1} \mathrm{H}-\mathrm{NMR}$ spectra of all the polymers were recorded on a Varian XL-200 $(200 \mathrm{MHz})$ or a Hitachi $\mathrm{H}-90$ spectrometer $(90 \mathrm{MHz})$.

The strength of the interaction between the acid- and base-polymers was evaluated from the $k_{\mathrm{AB}}$ value obtained by the polymer solvent method in viscosity measurement. ${ }^{3,4}$ The $k_{\mathrm{AB}}$ value reflects changes in molecular size of a polymer (A) due to the other polymer (B). For $1 / 3$ and $2 / 3$ pairs, the $k_{\mathrm{AB}}$ values were determined as follows. The acid-polymer $\mathbf{1}$ or $\mathbf{2}$ was dissolved in DMSO to give a series of solutions with concentrations ranging from $0.5 \times 10^{-3}$ to $2.0 \times 10^{-3} \mathrm{~g} \mathrm{~cm}^{-3}$. Then base-polymer 3 was dissolved in these "polymer solvents" to give a base-polymer concentration of $5.0 \times 10^{-3} \mathrm{~g} \mathrm{~cm}^{-1}$. After filtration, the solution viscosities were determined at $303 \pm 0.1 \mathrm{~K}$ by using a Ubbelode viscometer, and expressed in terms of intrinsic viscosities of the basepolymer (B) in the acid-polymer (A) solvent $\left(\left[\eta_{\mathrm{B}}\right]_{\mathrm{A}}\right)$. The $k_{\mathrm{AB}}$ values were evaluated using the following equation $^{3,4}$ :

$$
\left[\eta_{\mathrm{B}}\right]_{\mathrm{A}} \eta_{\mathrm{r}}\left(C_{\mathrm{A}}\right) /\left[\eta_{\mathrm{B}}\right]=1+2 k_{\mathrm{AB}}\left[\eta_{\mathrm{A}}\right] C_{\mathrm{A}}+\cdots
$$

where $\eta_{\mathrm{r}}\left(C_{\mathrm{A}}\right)$ is the relative viscosity of the acid-polymer at concentration $C_{\mathrm{A}}$, and $\left[\eta_{\mathrm{A}}\right]$ and $\left[\eta_{\mathrm{B}}\right]$ are the intrinsic viscosities of acid- and base-polymers in pure DMSO. In addition to $k_{\mathrm{AB}}$, Huggins coefficients $\left(k^{\prime}\right)$ were determined for each polymer at $303 \mathrm{~K}$ in DMSO and in methanol.

The UV spectra of the acid- and base-polymer mixtures were recorded on Hitachi U-3500, using a slit of $5 \mathrm{~nm}$, and quartz cells with the optical path length of $1 \mathrm{~mm}$ for DMSO, and $10 \mathrm{~mm}$ for methanol. The pyridyl concentrations of polymer 3 were $6 \times 10^{-3} \mathrm{moll}^{-1}$ for DMSO, and $3 \times 10^{-4} \mathrm{moll}^{-1}$ for methanol. DMSO and methanol were purified by distillation before use.

\section{RESULTS AND DISCUSSION}

Figure 1 and Table $I$ show the dependence of $\left[\eta_{B}\right]_{A}$ on $C_{\mathrm{A}}$ for $\mathbf{1} / \mathbf{3}$ and $\mathbf{2} / 3$ pairs. In both pairs, $\left[\eta_{\mathrm{B}}\right]_{\mathrm{A}}$ decreased very slightly with $C_{\mathrm{A}}$. This behavior may be assigned to the decrease in the dimensions of base-polymer in the 


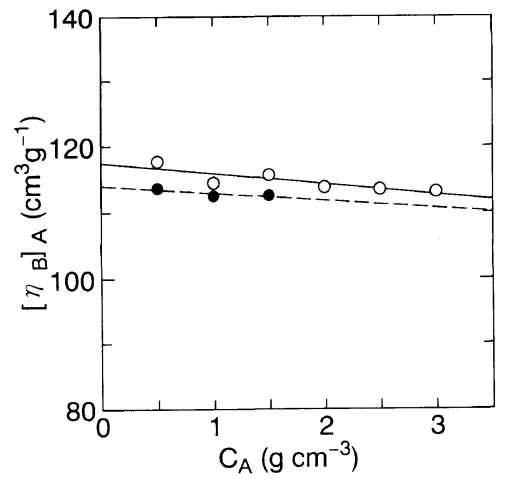

Figure 1. The dependence of $\left[\eta_{\mathrm{B}}\right]_{\mathrm{A}}$ on $C_{\mathrm{A}}$ for $\mathbf{1} / \mathbf{3}(\bigcirc, \longrightarrow)$ and $\mathbf{2} / \mathbf{3}$ $(\mathbf{0},--)$ pairs in DMSO.

Table I. Dependence of $\left[\eta_{\mathrm{B}}\right]_{\mathrm{A}}$ on $C_{\mathrm{A}}$

\begin{tabular}{ccc}
\hline & \multicolumn{2}{c}{$\left[\eta_{\mathrm{B}}\right]_{\mathrm{A}} / \mathrm{cm}^{3} \mathrm{~g}^{-1}$} \\
\cline { 2 - 3 }$C_{\mathrm{A}} \times 10^{3} / \mathrm{g} \mathrm{cm}^{-3}$ & $\mathbf{1}(\mathrm{A}) / \mathbf{3}(\mathrm{B})$ & $\mathbf{2}(\mathrm{A}) / \mathbf{3}(\mathrm{B})$ \\
\hline 0.5 & 117 & 114 \\
1.0 & 115 & 113 \\
1.5 & 116 & -113 \\
2.0 & 114 & - \\
2.5 & 114 & - \\
3.0 & 113 & \\
\hline
\end{tabular}

presence of acid-polymer. However, it was pointed out ${ }^{4}$ that the dependence of $\left[\eta_{\mathrm{B}}\right]_{\mathrm{A}}$ on $C_{\mathrm{A}}$ cannot be simply related to dimensional changes of base-polymer, and that $k_{\mathrm{AB}}$ value characterizes the acid-/base-polymer interaction better.

To get the $k_{\mathrm{AB}}$ values for $\mathbf{1} / \mathbf{3}$ and $\mathbf{2} / \mathbf{3}$ pairs, Figure 2 shows the plot of $\left[\eta_{\mathrm{B}}\right]_{\mathrm{A}} \cdot \eta_{\mathrm{r}}\left(C_{\mathrm{A}}\right) /\left[\eta_{\mathrm{B}}\right]$ against $\left[\eta_{\mathrm{A}}\right] C_{\mathrm{A}}$. These plots gave good linear relation. From the slopes of these lines, each $k_{\mathrm{AB}}$ value was calculated and shown in Table II. In addition, Table II shows the $k^{\prime}$ value of each polymer in DMSO and in methanol. Both the $k_{\mathrm{AB}}$ values were larger than every $k^{\prime}$ value in DMSO, indicating that the interactions between the acid- and base-polymers are stronger than those between the acidpolymers or between the base-polymers. The reason for this may be that the former interactions involve the hydrogen bonding between carboxyl and pyridyl groups in both polymers:

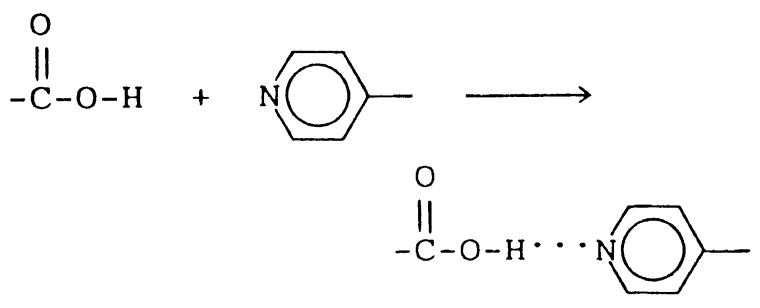

On the other hand, $\mathbf{1} / \mathbf{3}$ and $\mathbf{2} / \mathbf{3}$ pairs showed almost the same $k_{\mathrm{AB}}$ value. Thus, the acid-/base-polymer interactions in DMSO are almost the same strength in the two pairs.

Interactions in these systems will consist of van der Waals forces and hydrogen-bonding. However, hydrogen-bonding interactions are considered to be less effective for the interactions, since strong hydrogen-bond accepting nature of DMSO interferes the hydrogen-

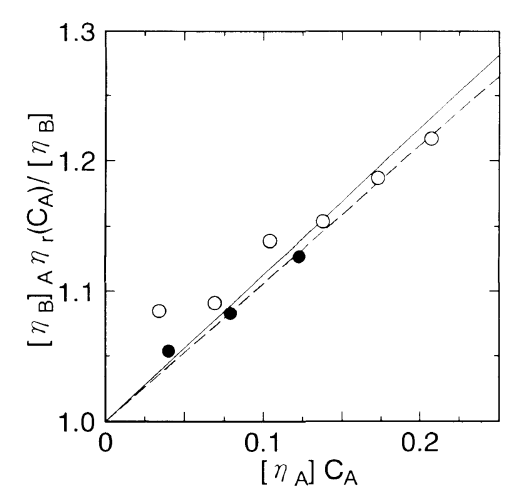

Figure 2. The plot according to eq 1 for $\mathbf{1} / \mathbf{3}(\bigcirc,-)$ and $\mathbf{2} / \mathbf{3}$ ( pairs in DMSO.

Table II. Interaction coefficients $\left(k_{\mathrm{AB}}\right)$ and Huggins coefficients $\left(k^{\prime}\right)$ at $303 \mathrm{~K}$

\begin{tabular}{ccccc}
\hline \multirow{2}{*}{$\begin{array}{c}\text { Polymer } \\
\text { pair }\end{array}$} & \multicolumn{2}{c}{$k_{\mathrm{AB}}$} & & \multicolumn{2}{c}{$k^{\prime}$} \\
\cline { 2 - 3 } \cline { 4 - 5 } & In DMSO & & In DMSO & In methanol \\
\hline $\mathbf{1} / \mathbf{3}$ & 0.56 & $\mathbf{1}$ & 0.37 & 0.53 \\
$\mathbf{2} / \mathbf{3}$ & 0.53 & $\mathbf{2}$ & 0.41 & 0.76 \\
& & $\mathbf{3}$ & 0.39 & 0.30 \\
\hline
\end{tabular}
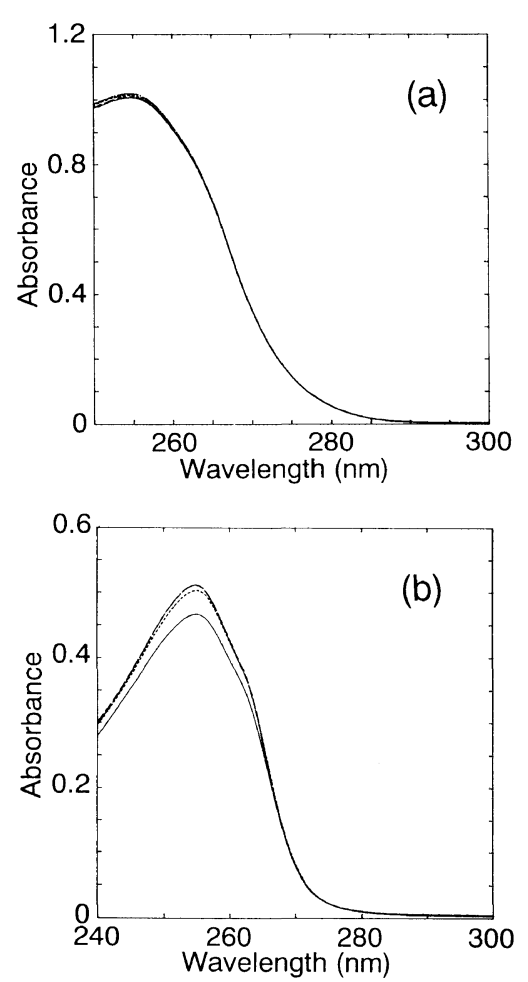

Figure 3. The effect of adding polymer 1 on UV spectra of polymer 3 (a) in DMSO ([pyridyl] $=6 \times 10^{-3} \mathrm{moll}^{-1}$ ) and (b) in methanol ([pyridyl] $\left.=3 \times 10^{-4} \mathrm{moll}^{-1}\right)$. $[\mathrm{COOH}] /[$ pyridyl $]: 0.0(-), 1.0$ $(---), 2.0(--)$, and $5.0(---)$.

bonding between carboxyl and pyridyl groups. In fact, the NMR spectra of acid-polymers 1 and 2 in $\left(\mathrm{CD}_{3}\right)_{2} \mathrm{SO}$ showed a carboxyl proton peak around $12.5 \mathrm{ppm}$, suggesting strong hydrogen bonding interaction between the acid-polymer and DMSO. ${ }^{5}$

Further, we measured UV spectra of acid- and basepolymer mixtures to know if hydrogen bonding occurs between carboxyl and pyridyl groups in DMSO. Figure 
3 shows effect of adding acid-polymer 1 on UV spectra of base-polymer 3. No marked changes in the UV spectra were observed for $\mathbf{1 / 3}$ pair as well as $2 / 3$ pair (data not shown). On the other hand, the absorption intensity of polymer $\mathbf{3}$ in methanol increased with adding polymer 1, as shown in Figure 3. This hyperchromic effect $^{6}$ is ascribed to the formation of hydrogen bonds between the carboxyl and pyridyl groups. Therefore, DMSO should interfere the hydrogen-bonding interaction between the acid- and base-polymers.

\section{CONCLUDING REMARKS}

In the present study, the strength of interaction, i.e., $k_{\mathrm{AB}}$, was estimated for the sequence-ordered acidcopolymer/base-homopolymer pair as well as the acid-homopolymer/base-homopolymer pair. Both pairs showed similar interactions in DMSO. This reason is mainly based on stronger hydrogen-bond accepting nature of DMSO: i.e., DMSO forms strong hydrogen bonding with acid-polymers, and thus weakens the hydrogen-bonding interaction between acid- and basepolymers. As a result, even if carboxyl groups in the acid-polymer interact with pyridyl groups in the base-polymer, the interactive carboxyl groups will appear partly and randomly along the acid-polymer chain. Namely, DMSO interferes the formation of consecutive hydrogen bonds between carboxyl and pyridyl groups. Consequently, the difference in the acid-polymer sequences did not reflect their polymer complexations in

\section{DMSO.}

The $k_{\mathrm{AB}}$ values in DMSO originate mainly from van der Waals forces (mainly, hydrophobic interaction) between the acid- and base-polymers, because DMSO weakens hydrogen-bonding interactions in interpolymer complexation and has highly polar nature (dielectric constant $=46.6$ ). The similarity in $k_{\mathrm{AB}}$ values for $1 / 3$ and $2 / 3$ pairs should indicate that both hydrophobic interactions have similar strength in polar solvent. Thus, the introduction of two hydrophobic ethylene units into acid-polymer sequence does not affect the degree of hydrophobic interaction.

Acknowledgment. This study is supported by a Giant-in-Aid for Cooperative Research (A), the Ministry of Education, Science, and Culture of Japan.

\section{REFERENCES}

1. K. Yokota, Kobunshi Kako, 44, 29 (1995).

2. Y. Inai, S. Kato, T. Hirabayashi, and K. Yokota, Polym. J., 27, 196 (1995).

3. N. Tewari and A. K. Srivastava, Macromolecules, 25, 1013 (1992)

4. D. Staszewska, J. Kovar, and M. Bobdanecky, Colloid Polym. Sci., 258, 600 (1980).

5. H. Ohno, K. Abe, and E. Tsuchida, Makromol. Chem., 179, 755 (1978).

6. R. M. Silverstein, G. C. Bassler, and C. M. Terence, "Spectrometric Identification of Organic Compounds," 4th ed, John Wiely \& Sons, New York, N.Y., 1981, Chapter 6. 